\title{
COMMENTARY
}

\section{Optimizing safe, comfortable ICU care through multi-professional quality improvement: just DO it}

\author{
Alison J Montpetit*1 and Curtis N Sessler ${ }^{2}$ \\ See related research by de Jong et al., http://ccforum.com/content/17/2/R74
}

\begin{abstract}
Translating research to the bedside can present significant challenges in the complex ICU environment. In this issue of Critical Care, de Jong and colleagues report on a quality improvement project (NURSEDO) that led to a decrease in severe pain and serious adverse events during nursing care procedures in their ICU. In this commentary we describe three aspects of this quality improvement study that we think contributed to the overall success of the NURSE-DO project: the hospital environment and culture; multiprofessional partnerships; and an evidence-based structured approach.
\end{abstract}

Translating research to the bedside can present significant challenges in the complex ICU environment, resulting in a lag between evidence and clinical practice, particularly in multifactorial issues such as pain management. Despite research acknowledging pervasive pain in critically ill patients and clinical practice guidelines recommending routine assessment and pre-emptive treatment, barriers still exist in treating pain with analgesics in the ICU. Most commonly, an underappreciation of pain, concern of serious adverse events, and lack of a structured approach prevent effective pain management in the ICU.

In this issue of Critical Care, de Jong and colleagues report on a quality improvement (QI) project (NURSEDO) that led to a decrease in severe pain and serious adverse events during nursing care procedures in their ICU [1]. In this commentary we describe three aspects of this QI study that we think contributed to the overall success of the NURSE-DO project: the hospital

\footnotetext{
*Correspondence: ajmontpetit@vcu.edu

'Department of Adult Health and Nursing Systems, Virginia Commonwealth University, School of Nursing, PO Box 980567, Richmond, VA 23298, USA Full list of author information is available at the end of the article
}

environmental and culture; multi-professional partnerships; and a structured approach.

\section{Hospital environment and culture}

We view this QI study as the third phase of the investigators' stepwise QI initiative, starting in 2002 with an assessment of pain and agitation [2], followed in 2006 with evaluation of sedation protocol-driven nursing interventions [3], and culminating in the current NURSEDO project that began in 2010 and evaluates the effect of a QI pain management process on patient outcomes [1]. It is logical that the investigators' continuous engagement, now spanning 10 years, plays a critical role in their success. Systematic, continuous engagement builds a foundation of knowledge and confidence in implementing QI projects among practitioners but, more importantly, conveys a clear message for the critical value of pain management and cultivates a hospital environment culture that is supportive of multi-professional QI initiatives [4].

\section{Multi-professional partnerships}

Owing to negative sequelae of oversedation, revised clinical practice guidelines for the treatment of pain in the ICU highlight the importance of a personalized, patient-centered approach that promotes comfort using the lowest effective dose of analgesic medication [5]. Nurses are on the frontline of patient care and are direct observers of pain behaviors and therapeutic responses; nurses are therefore key stakeholders in promoting safe comfortable care. However, organizations that support a shared responsibility for the quality of care - including nurses, physicians, and clinical pharmacists - are better positioned to achieve and sustain improvement $[4,6]$.

Although entitled NURSE-DO, the approach for this study appears to be 'We-DO' where nurses, physicians, nurse assistants, nurse managers and medical directors were all engaged at various stages of the process, sharing responsibility [1]. This is highlighted in activities during the 'adjust' step, where medical staff adjusted orders, nurses had more choices related to analgesics, and administrators were involved in weekly rounds. In the 
following phase 3 evaluation, significant differences were observed with a decrease in severe pain and in serious adverse events, and an increase in analgesic use. Although we cannot presume the exact cause for the significant differences, we can hypothesize that multiprofessional involvement and the increase in nurse autonomy may have contributed. Sustainable success in QI initiatives (with multi-professional involvement) is increased when expectations are set for all staff [4].

\section{Structured approach}

Use of a multi-professional, structured approach that aligns with clinical practice guidelines [5] is important in the management of analgesia and successful QI implementation [7]. This concept was central in this study, beginning with the framework Plan-Do-Check-Adjust, an effective system for QI project implementation. The concept was also mirrored in the evidence-based approach to pain management, beginning with systematic evaluation of pain utilizing reliable, valid tools, preemptive treatment of pain prior to nursing care procedures, escalation in analgesic use based on the World Health Organization's pain relief ladder, and inclusion of nonpharmacologic interventions. In the NURSE-DO study, the investigative team provided ongoing feedback in the 'adjust' step and throughout the study. This included visual reminders, necessary equipment and study flowsheets in each patient room, and engagement of unit leadership during weekly rounds - all of which are shown to increase success in QI [4]. A multi-professional, stepwise approach is imperative in improving pain management while avoiding oversedation and unnecessary side effects.

Variation in the appreciation of pain and concern that analgesics might cause harmful side effects are common issues among practitioners. This variation is probably influenced by the extent of patient contact, care-provider educational background, previously witnessed negative outcomes, and the critical conditions inherent in the ICU population. However, the safe management of pain in the ICU is a necessary, albeit critically complex, issue to address. A structured approach builds upon objective measures and employs a hierarchy of interventions, reducing variation. The NURSE-DO project convincingly illustrates that successful QI initiatives are attainable despite the complex ICU environment, through a stepwise, team-based approach within the context of a supportive hospital environment culture. As our ICU practice moves inexorably towards even earlier and more dramatic mobilization - potentially with added discomfort, stress, and risk - we must build upon the core elements of programs such as the NURSE-DO project to achieve rapid recovery that is safe and minimizes pain.

Abbreviations

Ql, quality improvement.

\section{Competing interests}

The authors declare that they have no competing interests.

\section{Acknowledgements}

AJM and CNS are supported by the National Institute of Nursing Research (R00 NR012016).

\section{Author details}

'Department of Adult Health and Nursing Systems, Virginia Commonwealth University, School of Nursing, PO Box 980567, Richmond, VA 23298, USA

${ }^{2}$ Department of Internal Medicine, Division of Pulmonary Disease and Critical Care Medicine, Virginia Commonwealth University, School of Medicine, PO Box 980050, Richmond, VA 23298, USA.

Published: 30 April 2013

References

1. de Jong A, Molinari M, de Lattre S, Gniadek C, Carr J, Conseil M, Susbielles M-P, Jung B, Jaber S, Chanques G: Decreasing severe pain and serious adverse events while moving intensive care unit patients: a prospective interventional study (the NURSE-DO project). Crit Care 2013, 17:R74.

2. Chanques G, Jaber S, Barbotte E, Violet S, Sebbane M, Perrigault PF, Mann C, Lefrant JY, Eledjam JJ: Impact of systematic evaluation of pain and agitation in an intensive care unit. Crit Care Med 2006, 34:1691-1699.

3. Dodek P, Chanques G, Brown G, Norena M, Grubisic M, Wong H, Jaber S: Role of organisational structure in implementation of sedation protocols: a comparison of Canadian and French ICUs. BMJ Qual Saf 2012, 21:715-721.

4. Draper DA, Felland LE, Liebhaber A, Melichar L: The role of nurses in hospital quality improvement. Res Brief 2008, 3:1-8.

5. Barr J, Fraser GL, Puntillo K, Ely EW, Gélinas C, Dasta JF, Davidson JE, Devlin JW, Kress JP, Joffe AM, Coursin DB, Herr DL, Tung A, Robinson BR, Fontaine DK, Ramsay MA, Riker RR, Sessler CN, Pun B, Skrobik Y, Jaeschke R; American College of Critical Care Medicine: Clinical practice guidelines for the management of pain, agitation, and delirium in adult patients in the intensive care unit. Crit Care Med 2013, 41:263-306.

6. Sessler CN: Progress toward eliminating inadequately managed pain in the ICU through interdisciplinary care. Chest 2009, 135:894-896.

7. Pasero C, Puntillo K, Li D, Mularski RA, Grap MJ, Erstad BL, Varkey B, Gilbert HC, Medina J, Sessler CN: Structured approaches to pain management in the ICU. Chest 2009, 135:1665-1672.

doi:10.1186/cc12601

Cite this article as: Montpetit AJ, Sessler CN: Optimizing safe, comfortable ICU care through multi-professional quality improvement: just DO it. Critical Care 2013, 17:138. 